\title{
Lorenz model and chaos masking /addition technique
}

\author{
Dina Ahmed Kafi, Raied Kamel Jamal, K. A. Al- Naimee \\ Department of Physics, College of Science, University of Baghdad, Baghdad, Iraq \\ E-mail: dina.hanoo83@gmail.com
}

\begin{abstract}
In the present work, the chaotic signal that produces experimentally was studied by using Lorenz model. Nonlinear behavior (chaotic) in a nonlinear device was created using a Lorenz circuit. The dynamical system can be perceived by using the bifurcation diagram. In order to encode the message to the chaotic carrier signal, Lorenz circuit has been used. The decoding of the message is achieved by the synchronization between the chaotic oscillator of the transmitter and the receiver.

This work studied the change of time series of the chaotic signal with changing the initial conditions where the stochastic parameters are $\sigma=10, \mathrm{R}=30$, and $\mathrm{b}=8 / 3$, while initial conditions are $\mathrm{X}_{\text {int }}=\mathrm{y}_{\text {int }}=\mathrm{Z}_{\text {int }}=0.01, \mathrm{x}_{1}=0.03$, and $\mathrm{y}_{\text {int }}=\mathrm{Z}_{\text {int }}=0.01$.
\end{abstract}

Key words

Chaotic signals, synchronization, master and slave, Lorenz circuits, optical fiber.

\section{Article info.}

Received: Jan. 2016

Accepted: Apr. 2016

Published: Dec. 2016

\section{نموذج لورنز و تقنية اخفاء/ اضافة الثواش

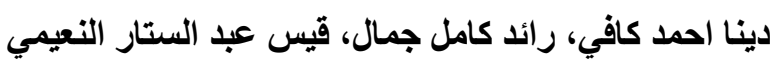 \\ قسم الفيزياء، كلية العلوم، جامعة بغداد، بغداد، العراق اقديأ}

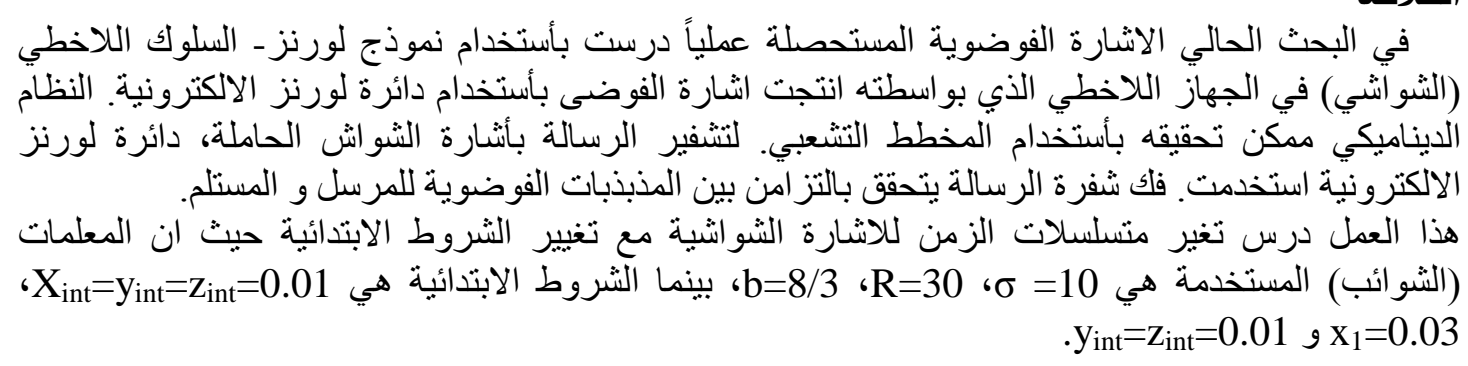

\section{Introduction}

Numerically Berkeley Madonna and Origin Lab software are used to graph and analyze the time series that generated in chaos regime. Moreover the analyses concern the study of attractor and the Fast Fourier Transformations FFT of the output spectrum of the Lorenz circuits has been demonstrated also. Finally bifurcation diagram of the Lorenz model was showed [1].

In secure communication the synchronization of the chaotic signal between the transmitter and the receiver is examined by using coupling factor [2]. The recovery of the message at the end of the receiver is investigated, which is based on the synchronization of the chaotic oscillator of the transmitter and the receiver [3]. In this study, the experimental of chaos by Lorenz model will be achieved. Effect of the initial condition value for $\mathrm{x}$ parameter has been demonstrated [4].

\section{The numerical model results}

The modeling approach of chaos was investigated by programming the 
physical model, where the total simulation time chosen depends strongly on the magnitude of the temporal scales defined by three parameters $\mathrm{R}, \sigma, b$, and also on initial conditions [5]. To demonstrate the first property of chaos, i.e. periodicity, a numerical simulation was done using Berkeley Madonna software of ordinary differential equations, (Eq. (1) below). To find the time series of $\mathrm{x}, \mathrm{y}$, and $\mathrm{z}$ the numerical simulation procedure was done by using the forth order Runge -Kutta method [6]. The stochastic parameters are $\sigma=10, \mathrm{R}=30$, and $b=8 / 3(2.66)$, while the initial conditions are $\mathrm{x}_{\text {int }}=0.01, \mathrm{y}_{\mathrm{int}}=0.01$, and $\mathrm{z}_{\text {int }}=0.01$.

The Lorenz system is defined by the system of ordinary differential equations [7]:

$$
\left.\begin{array}{l}
\mathrm{dx} / \mathrm{dt}=-\sigma \mathrm{x}+\sigma \mathrm{y} \\
\mathrm{dy} / \mathrm{dt}=\mathrm{Rx}-\mathrm{y}-\mathrm{x} \\
\mathrm{dz} / \mathrm{dt}=-b \mathrm{z}+\mathrm{xy}
\end{array}\right\}
$$

These equations in Lorenz model describing the fluid motion and heat flow.

where $\mathrm{x}, \mathrm{y}$, and $\mathrm{z}$ are the states of the system and $\sigma, R$, and $b$ are positive parameters (where $\sigma$ is the Prandtl number, $\mathrm{R}$ is the Rayliegh number and $\mathrm{b}$ is a geometric factor), which denote physical characteristics of air flow[9]. Typical parameter values that yields chaotic dynamics are $\sigma=10, R=30$, and $b=8 / 3=(2.66)$. For these values, the attractor generated by numerically integrating Eq. (1) is shown in Fig. 1. Typical waveforms for the three dynamical states are shown in Fig.2. To obtain a practical circuit design for the Lorenz system, it is necessary to scale the system equations.
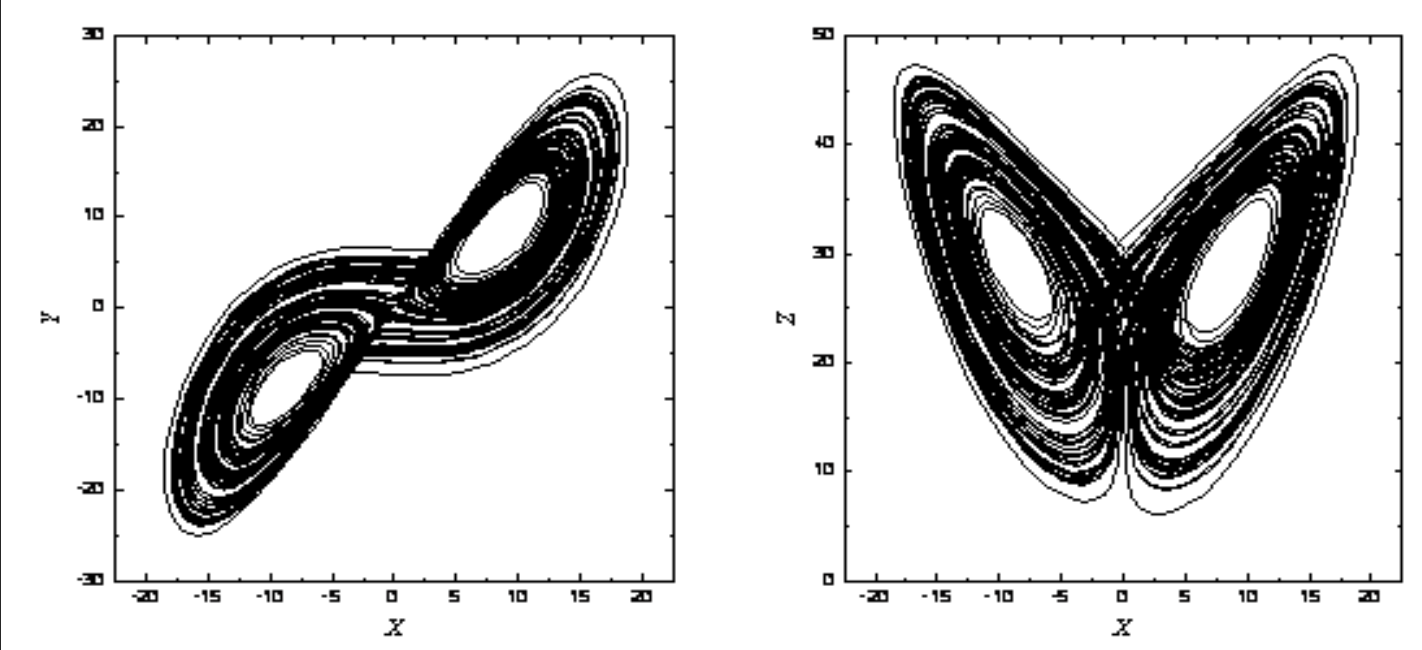

Fig.1: Lorenz attractor generated by numerical integration of the system equations with $\sigma=10, R=30$, and $b=8 / 3[10]$. 


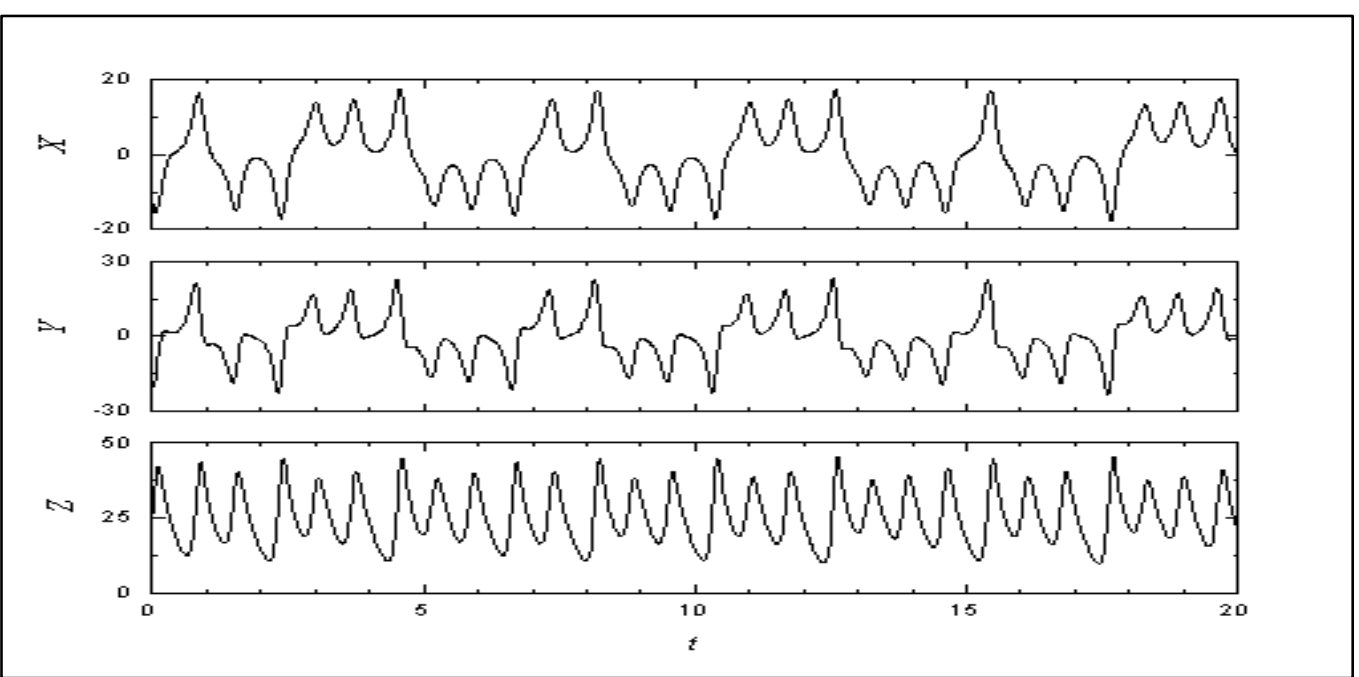

Fig.2: Typical Lorenz waveforms generated by numerical integration of the system equations with $\sigma=10, R=30$, and $b=8 / 3[9]$.

\section{Communication setup}

The communication setup includes the transmitter, receiver Lorenz circuits and synchronization circuit connecting them, as represented in Figs. 3 and 4. The resistors in Lorenz circuits were used as follows: $\mathrm{R}=1 \mathrm{k} \Omega, \mathrm{R}_{1}=75 \mathrm{k} \Omega$,
$\mathrm{R}_{2}=1 \mathrm{k} \Omega, \quad \mathrm{R}_{3}=7.5 \quad \mathrm{k} \Omega, \quad \mathrm{R}_{4}=28 \mathrm{k} \Omega$, $\mathrm{R}_{5}=2.9 \mathrm{k} \Omega, \mathrm{R}_{\mathrm{a}}=(0-3000) \mathrm{k} \Omega, \mathrm{R}_{\mathrm{g}}=(0-$ 3000) $\mathrm{k} \Omega, \mathrm{R}_{\mathrm{e}}=(0-2) \mathrm{k} \Omega$, and the capacitor C1 is $500 \mathrm{pF}$. The operational amplifiers $I_{1}, I_{2}$, and $I_{3}$ were of LF353 type, whereas the multipliers were of AD632 AD type.

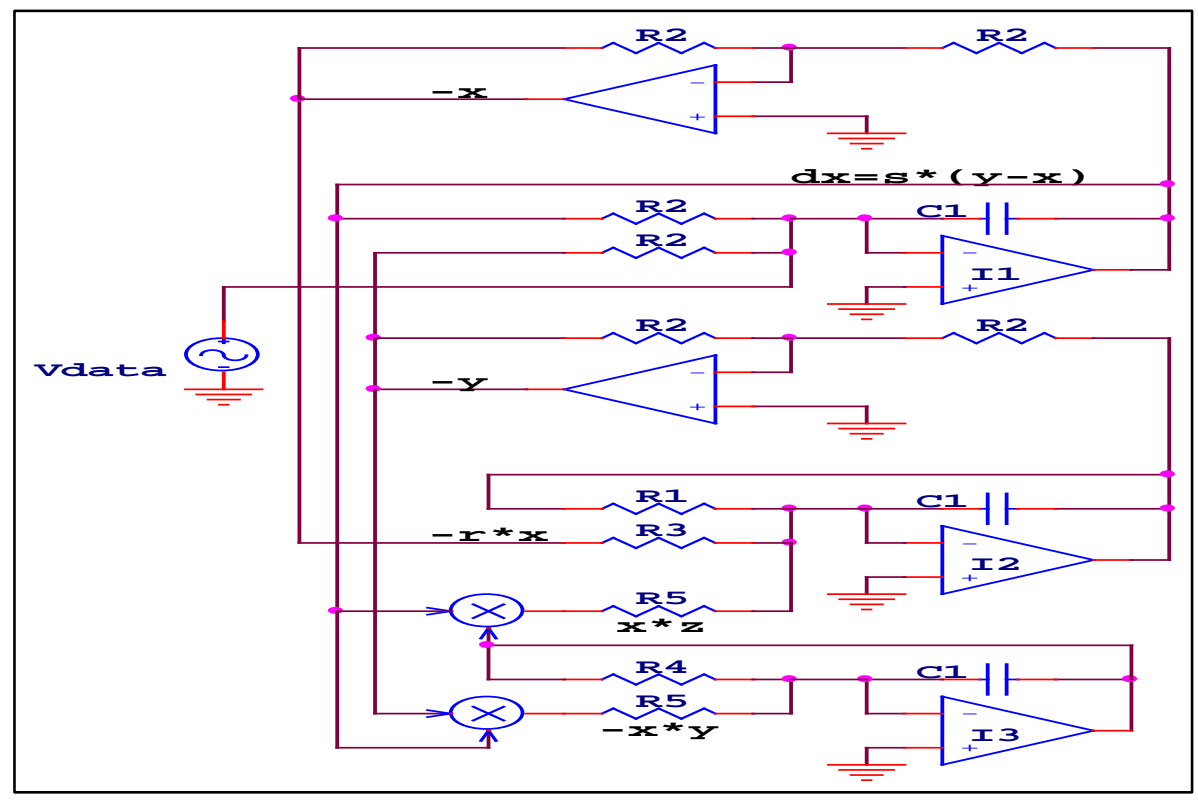

Fig.3: Lorenz circuit diagram. 


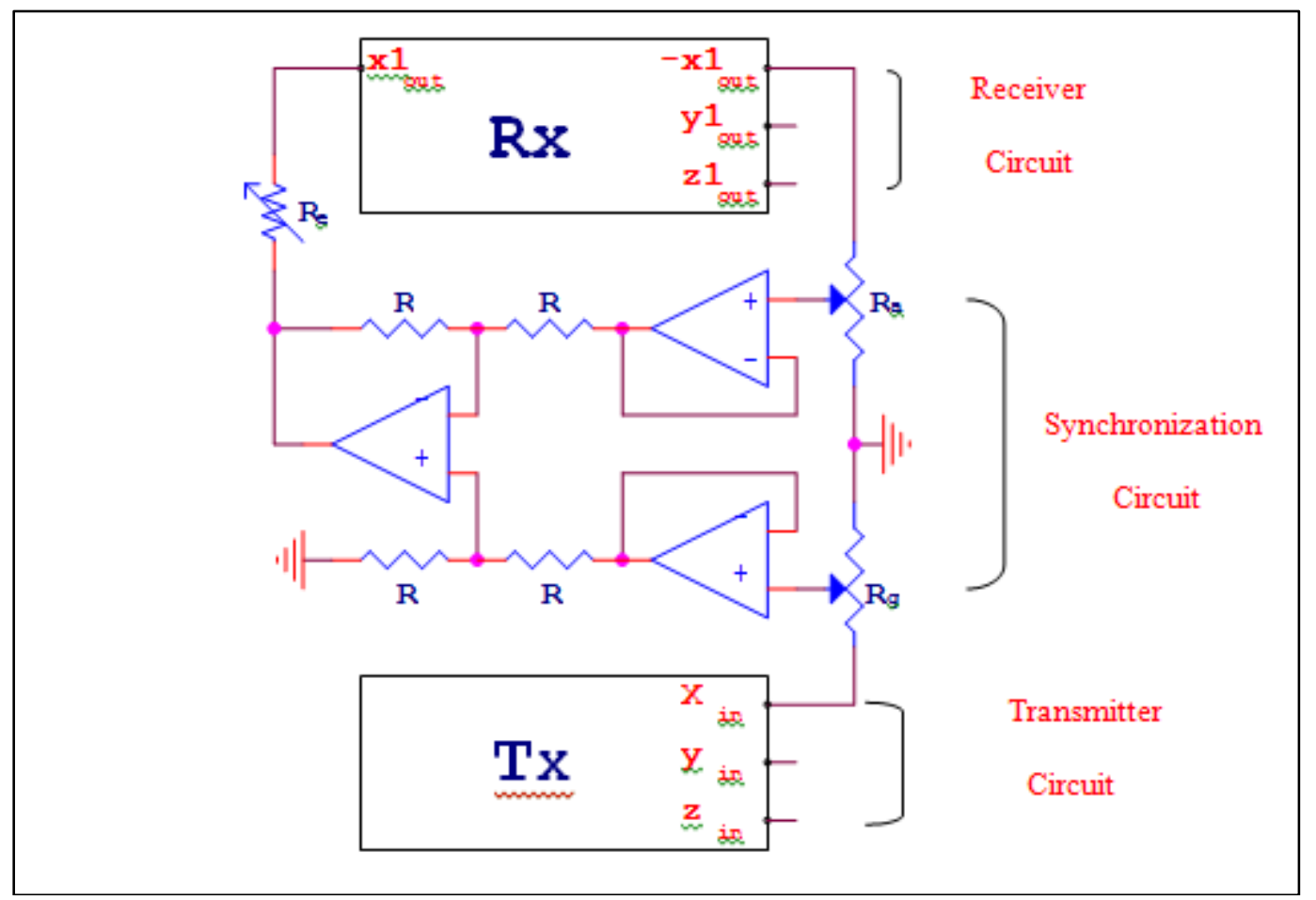

Fig.4: Synchronization circuit diagram (Lorenz model).

\section{Experimental results}

In secure communication, the massage that generated in an arbitrary waveform is modulated with chaotic signal that generated in transmitter Lorenz circuit $\left(\mathrm{T}_{\mathrm{r}}\right)$ as shown in Fig.5, the carried massage drive to electrooptical converter (E/O), where the carried massage convert from electrical massage to optical massage and drive it to semiconductor laser source. The semiconductor laser has continuous spectrum, but after adding the modulated signal to laser source the spectrum of laser take the shape of modulated signal (chaotic signal).

The message (perturbation frequency) could be recover by detected optical-chaos signal (O/E) converter and the synchronization must be happened by the received signal and the local chaos generated signal at the receiving part Fig.5 The synchronization has been made by changing the coupling strength gradually Fig.4. In complete synchronization condition, the message would be extracted and observed.

By applying voltage of the power supply $\pm 6.5 \mathrm{~V}$ on the electrical Lorenz circuit, the periodic oscillation of the dynamic behavior of the chaotic oscillator in $\mathrm{x}, \mathrm{y}$ and $\mathrm{z}$ direction shown in Figs.6, 7 and 8 respectively. The dynamics of the physical system under investigation depend on magnitude of the changed bias current, because all other parameters are constant. The constant control parameters are amplifier gain and capacitors value. 


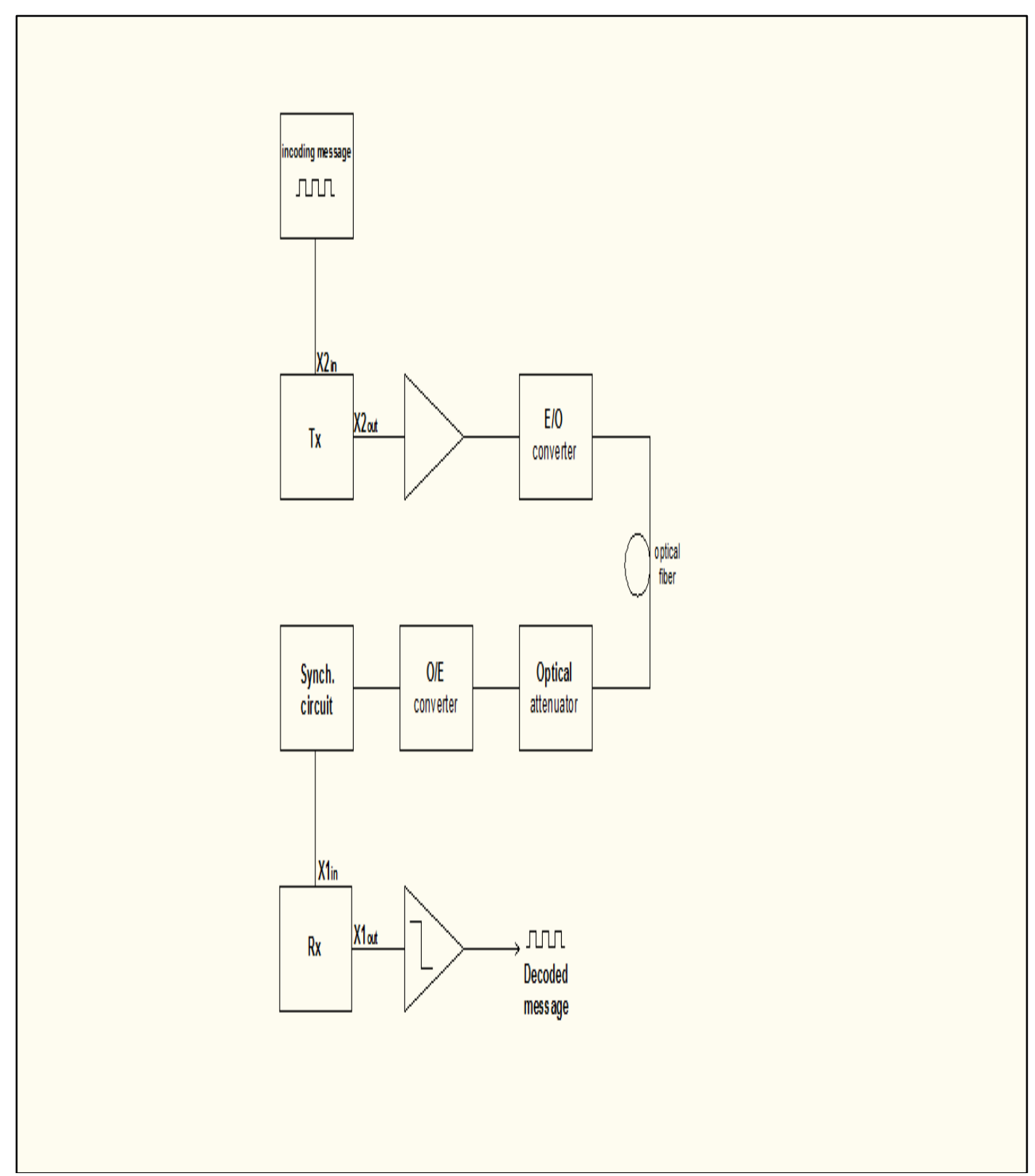

Fig.5: Optical communication system (Lorenz model).

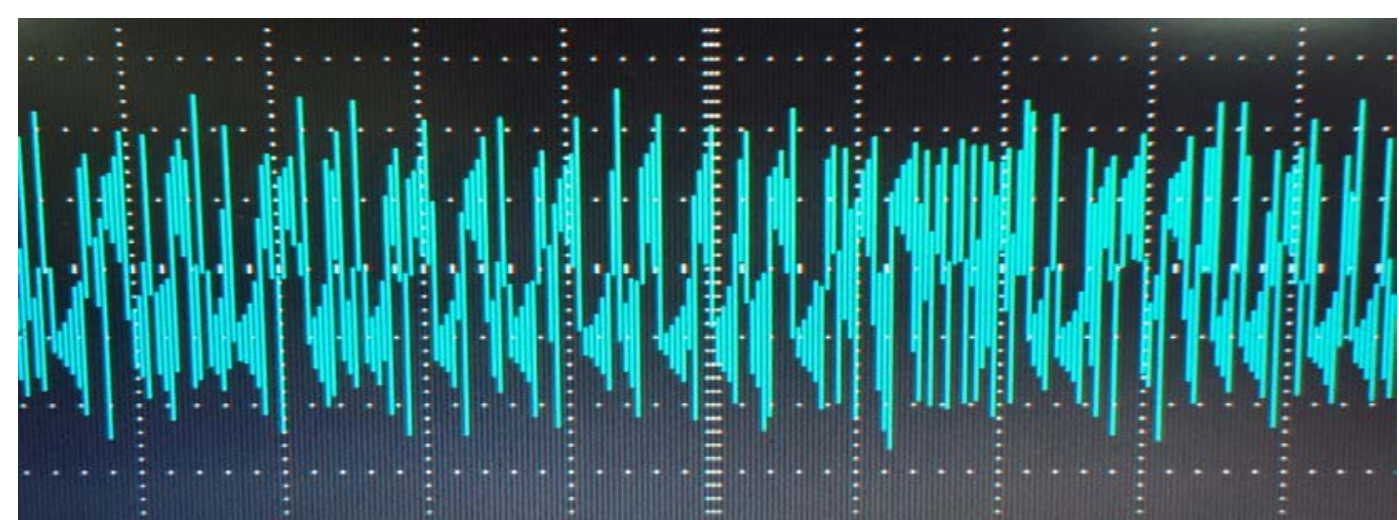

Fig.6: The intensity of $x$ dynamical state as function of time series. 


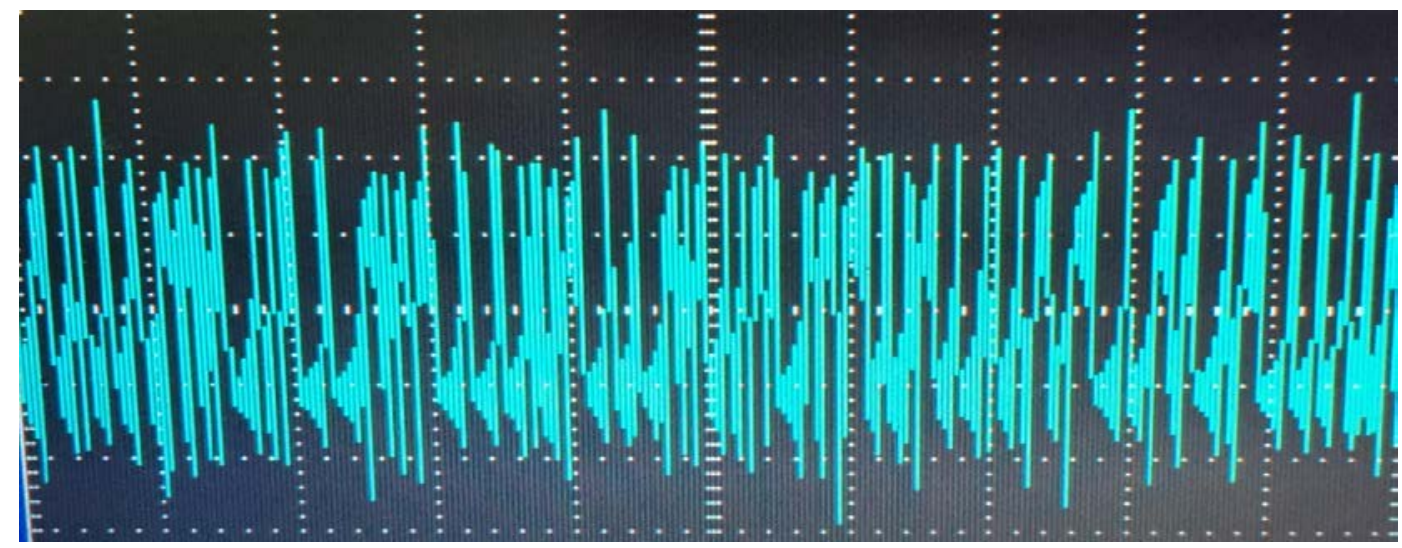

Fig.7: The intensity of y dynamical state as function of time series.

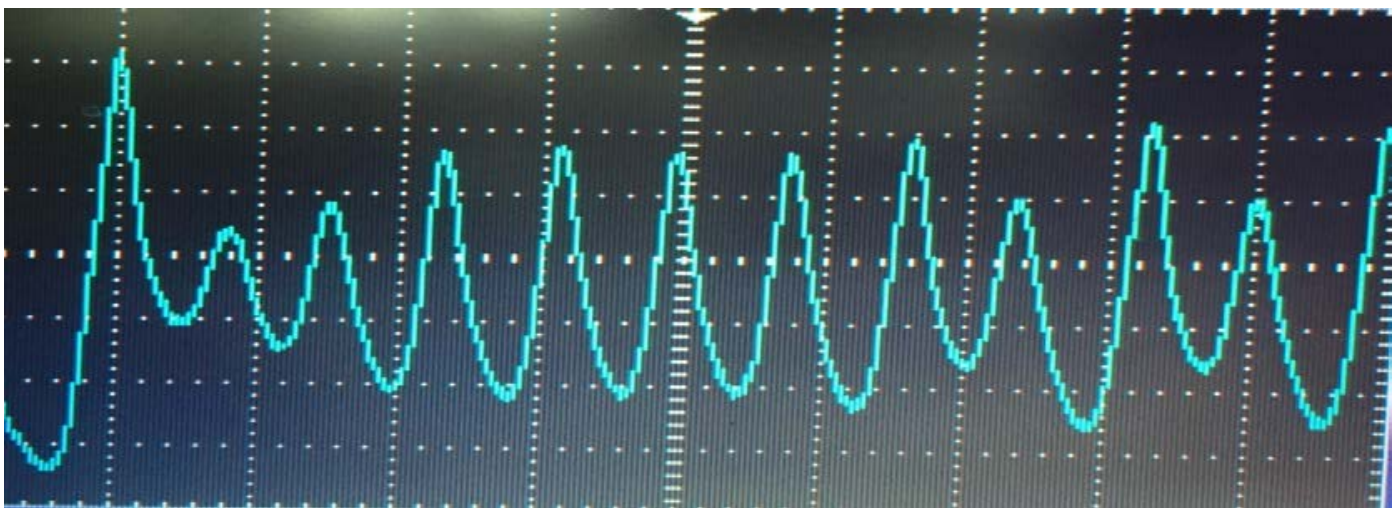

Fig.8: The intensity of $\mathrm{z}$ dynamical state as function of time series.

The phase space of the Lorenz attractor is mapped in minimal three dimensions required for continuous time chaos by the three computed states $\mathrm{x}, \mathrm{y}$, and $\mathrm{z}$. The famous "Owl face" or "butterfly" diagram of the
Lorenz attractor is produced by neglecting the y state and plotting the $\mathrm{z}$ and $\mathrm{x}$ states in two dimensions. This is shown in the oscilloscope picture Fig.9.

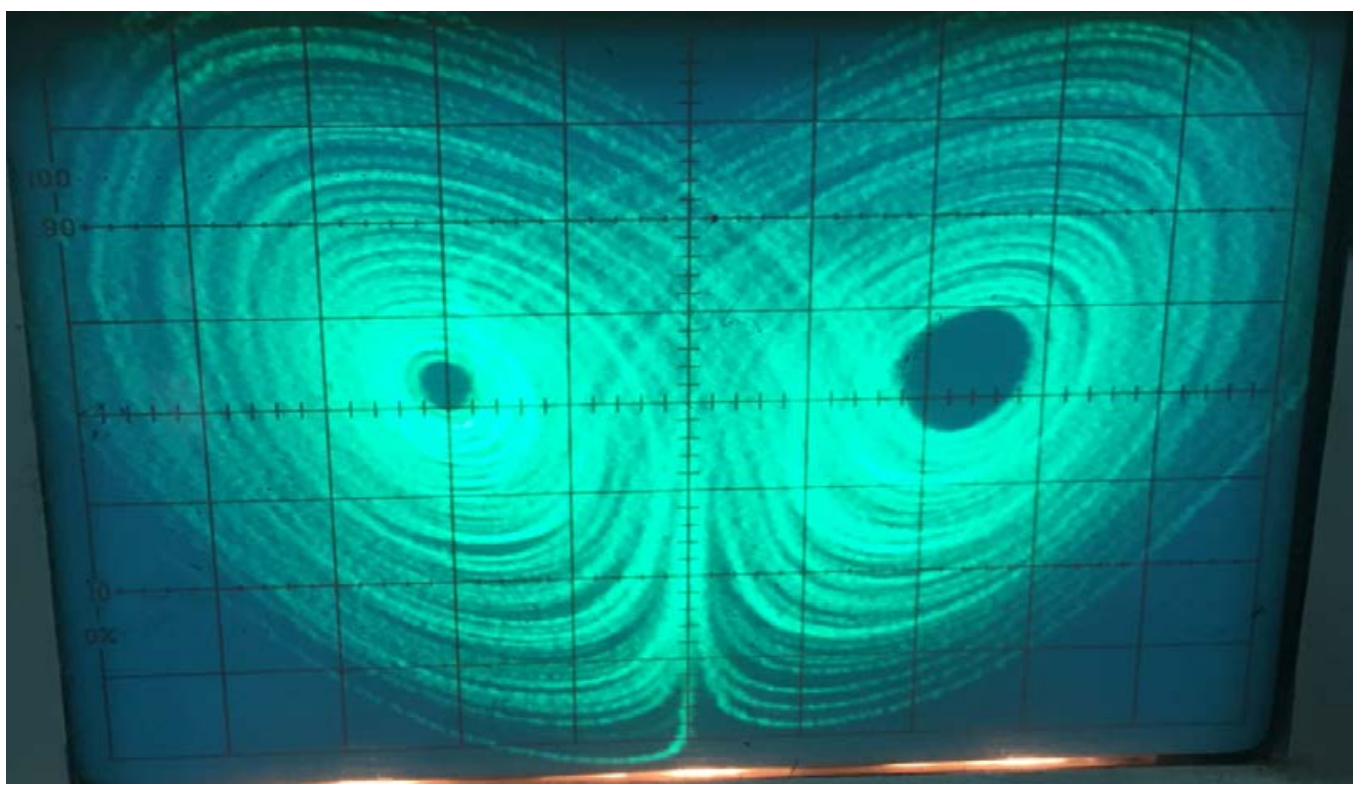

Fig.9: Strange attractor of the Lorenz Butterfly $\mathrm{z}$ against $x$ by numerical integration of the system equations. 
The $\mathrm{x}$ state is plotted on the horizontal axis and the $\mathrm{z}$ state on the vertical. It can be observed period doubling. To study the dynamics behavior of the chaotic oscillator of the transmitter under certain conditions, the bifurcation diagram should be plotting as shown in Fig.10. To get this diagram changing the value of the bias current in each try is essential to get many signals. The bifurcation diagram is constructed using peaks of the output signal intensity as $\mathrm{Y}$-axis versus the output signal as $\mathrm{X}$-axis, bifurcation control parameter. Time traces are recorded for resistance longing from $0 \Omega$ up to3000 $\mathrm{k} \Omega$, with $10 \Omega$ increments. At the resister of circuit $\left(0.25 \times 10^{3}\right) \mathrm{k} \Omega$, the oscillation begins as shown in Fig.5, where these oscillations are periodic. Sequentially, the resistance of circuit at $\left(0.5 \times 10^{3}\right) \mathrm{k} \Omega$, the system transition to doubling periodic. Finally at $\left(0.75 \times 10^{3}\right) \mathrm{k} \Omega$ the system gradually moves to chaotic system. The optimum value of resistor to get chaotic behavior was $2.7 \times 10^{3} \mathrm{k} \Omega$.

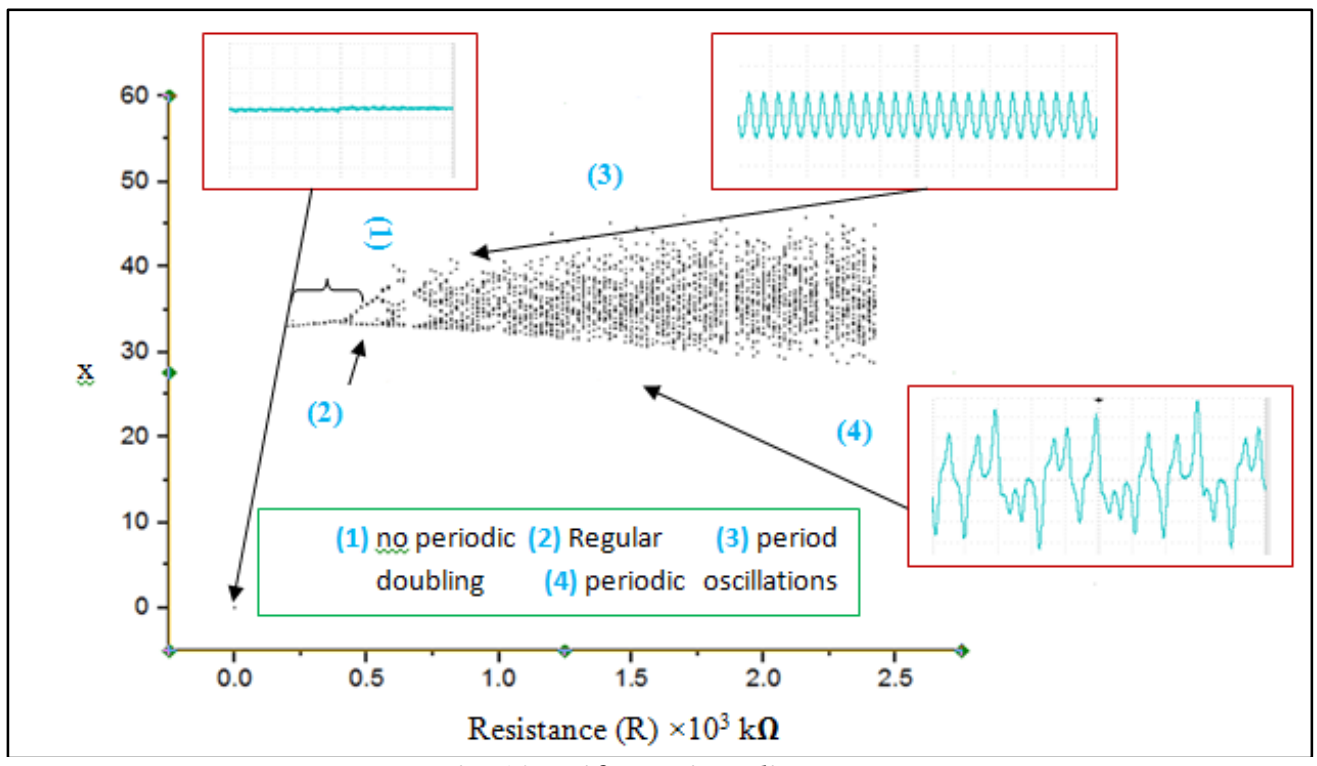

Fig.10: Bifurcation diagram.

\section{Communication systems}

From the bifurcation diagram, the best region for chaotic system is between $\left(2.5-2.7 \times 10^{3}\right) \mathrm{k} \Omega$, because the peak of amplitude of the chaotic carrier is very high at this region. The trajectories of two identical chaotic system that begin from slightly different initial condition quickly diverge and become uncorrelated even through each system can describe the same attractor in phase space. This phenomenon of synchronization is realized by uncoupling the two system, or in other words, by allowing fallow of information from the transmitter to the receiver. Our measurements are based on configuration setup that has been given in Fig.5. The signal of Fig. 11 was used in the transmitter unit and received in the receiver unit. Fig.12 shows the synchronization between the peaks amplitude of the transmitter and receiver. 


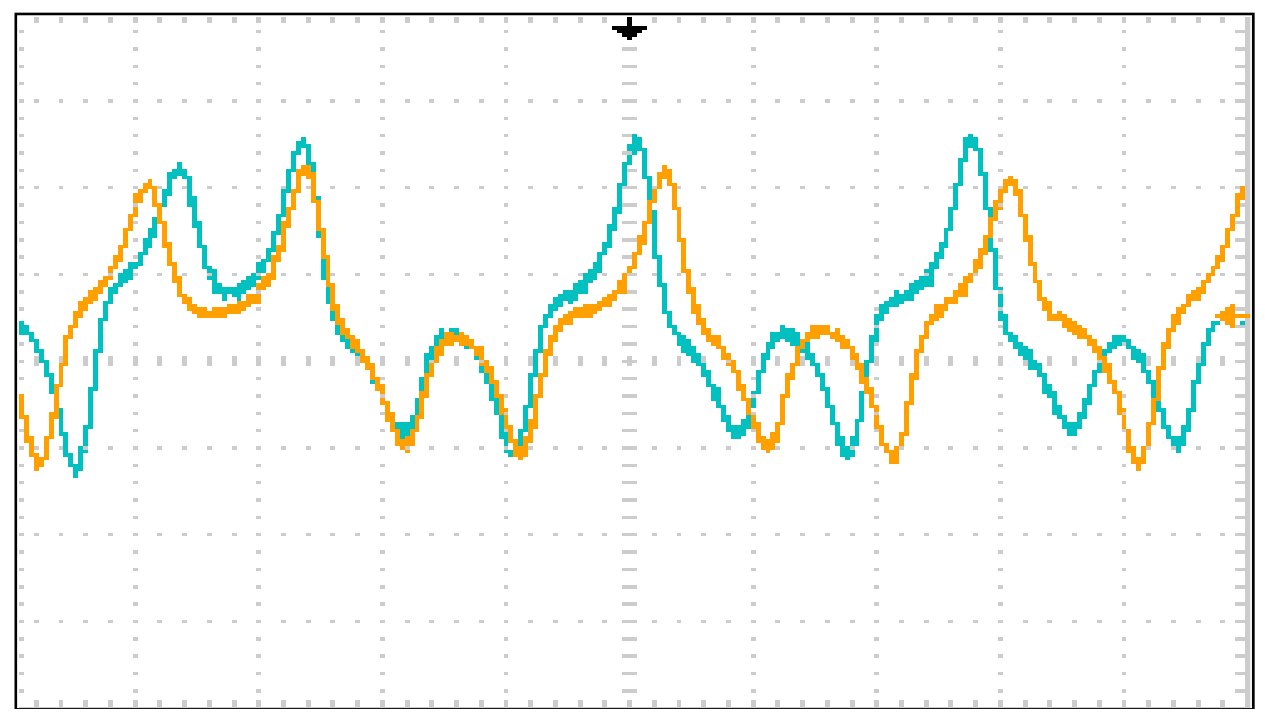

Fig.11: Time series of variable $x$ and $x_{1}$ of two similar Lorenz circuits' transmitter and receiver.

Full synchronization is obtained when the relation between the peaks amplitude of the transmitter and the receiver is a line at $45^{\circ}$ angle. The deviation from this line means an error in the synchronization between the transmitter and receiver. The massage is modulated with laser of wave length (850) nm at threshold current of (1550) $\mathrm{mA}$. The massage has $2 \mathrm{kHz}$ frequency and $100 \mathrm{mV}$ amplitude. The spectrum of chaotic signal and massage is given in Fig.13 where this signal would be attenuated when one work with security as in our job. The optical attenuator is placed before the modulated optical signal. In chaos modulation decryption technique, the message will be within the oscillators of the transmitter and the receiver as shown in Fig.13.

By using chaos masking decryption, Fig.14 showed the reference signal and chaotic oscillator receiver (replicated signal) output. While Fig.15 shows the recovery message by subtracting the reference signal and the replicated signal. By this way chaotic optical communication is a promising both privacy and security in communication networks.

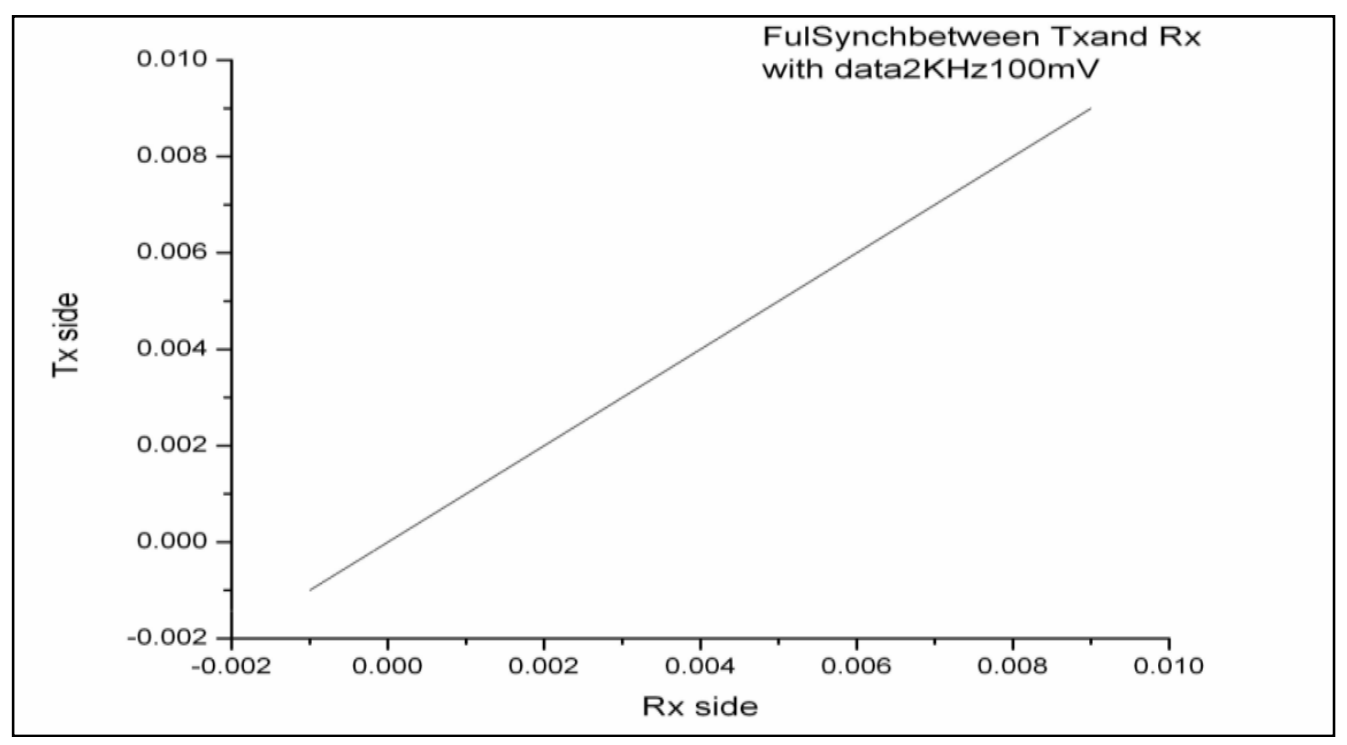

Fig.12: Full synch. between transmitter and receiver. 


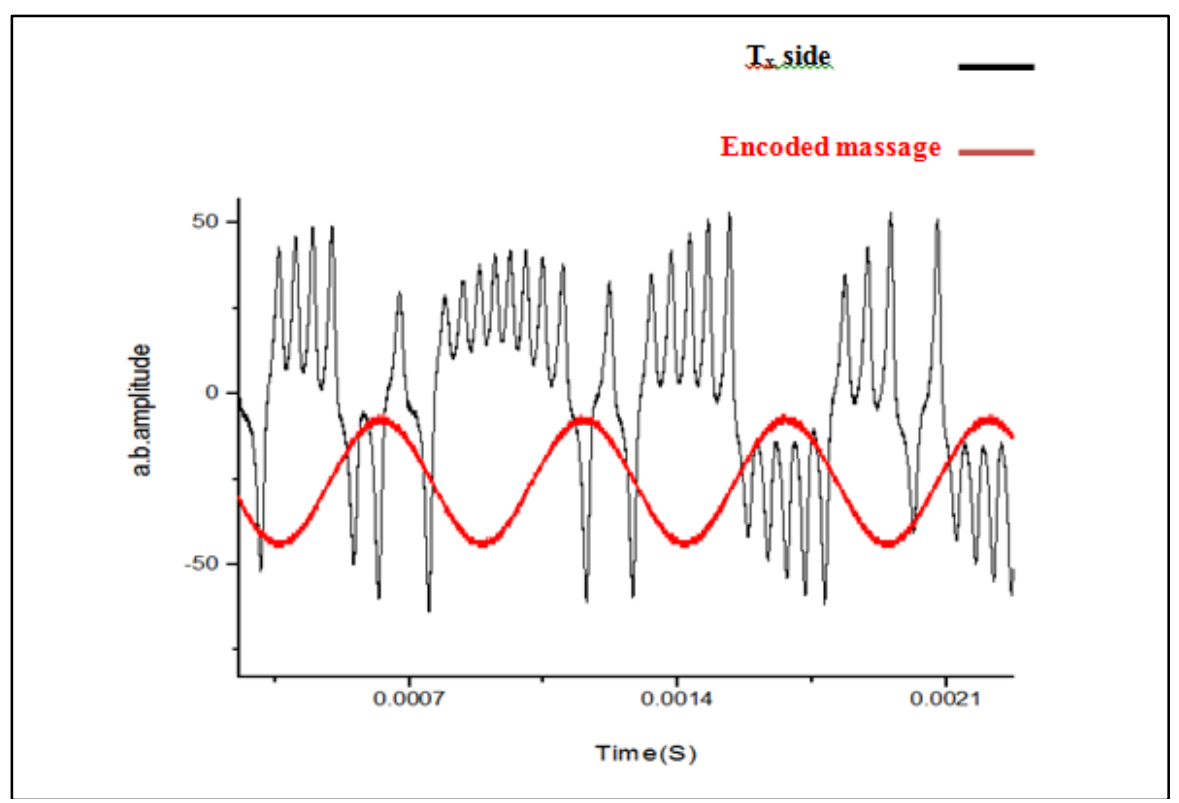

Fig.13: the encoded massage with data $2 \mathrm{kHz}$ frequency, and $100 \mathrm{mv}$ amplitude.

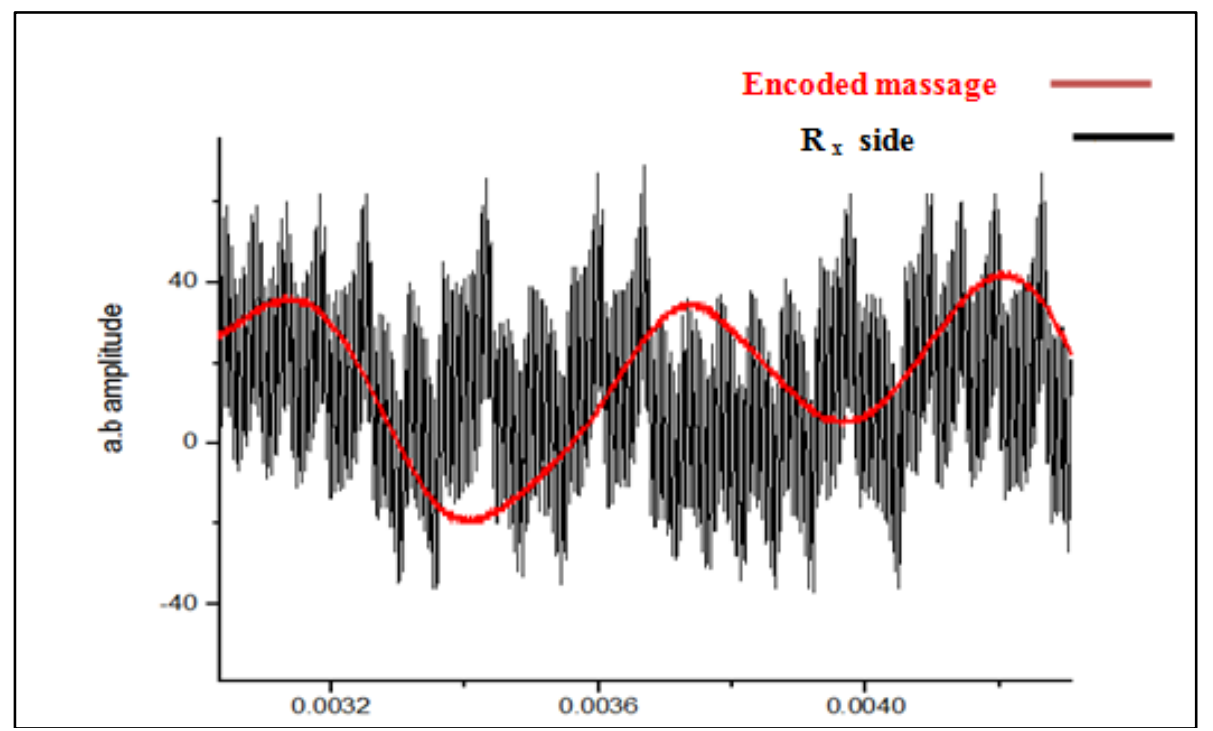

Fig.14: Rx chaotic signal with data $2 \mathrm{kHz}, 100 \mathrm{mv}$.

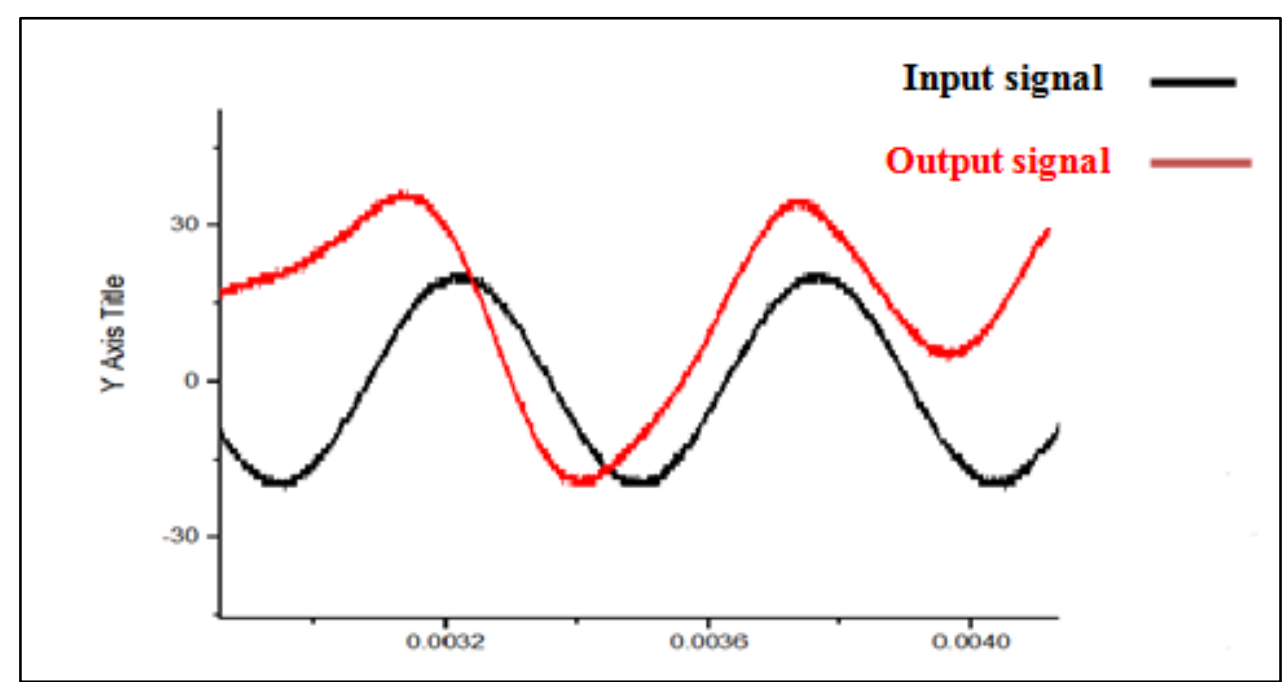

Fig.15: Input signal and output signal. 


\section{Conclusions}

This work demonstrates the feasibility of a simple method to synchronize two Lorenz systems with different parameters and then extend it to the recovery of the information signal, which is originally inserted into a chaotic signal, with satisfactory precision. The bifurcation diagram is an important to study the history of the system and to decide at which value of the $\mathrm{Rh}$ values is the best to modulate the message. The synchronization of this system is investigated. This method is easily implemented and also proved to be effective through numerical experiment. However it should be noted that the frequency of the masked information signal must be out of the range of the main frequency of the masking signal. To keep the communication more secure, the peak of power spectrum of the information signal had better be as indistinguishable from these of the neighboring frequencies of the masking variable as possible, that is" almost hidden "in the spectrum of the transmitted signal.

References
[1] E. Atlee Jackson, Perspectives of Nonlinear Dynamics, Cambridge University Press, New York (1990).

[2] C. R. S. Murthy, G. Srilatha, K. Anitha, Ch. Ravi Kumar, M. Srinivasa Rao, IJECT 2, 3, Sept. (2011) 12-14.

[3] W. Kinzel, A. Englert, I. Kanter, (On Chaos Synchronization and Secure Communication), Phil. Trans. R. Soc. A (2010) 368.

[4] K. Al-Naimee, F. Marino, M. Ciszak, R. Meucci, Arecchi T.F. New J. Phys., 11, 7 (2009) 73022.

[5] E. N. Lorenz, J. Atoms. Sci. 20, 2 (1963) 130-141.

[6] D. Kanakidis, A. Bogris, A. Argyris, D. Syvridis, IEEE J. Lightwave Technol., 22, 10 Oct. (2004) 2256-2263.

[7] C. Sparrow. The Lorenz equations, Bifurcations, Chaos and Strange atracttors. Springer, Verlage, (121128) 1982.

[8] T. L. Carroll and L. M. Pecora, IEEE Trans. Circuits Syst. 38, Apr. (1991) 453-456.

[9] K. M Cuomo, IEEE, 40 (1993) 10. [10] Kathy Ludge, (Nonlinear Laser Dynamics: from Quantum Dots to Cryptography), Wiley-VCH Verlag \& Co., 2012. 